\title{
RING OF ENDOMORPHISMS OF A FINITE LENGTH MODULE
}

\author{
R. N. GUPTA AND SURJEET SINGH
}

\begin{abstract}
An example of a uniserial module $M_{R}$ of composition length 2, such that $S=\operatorname{End}\left(M_{R}\right)$ acting on the left is not right artinian, is given. An elementary proof of a known result, that the ring of endomorphism of a finite length quasi-injective module $M_{R}$ acting on the left is left artinian, is also given.
\end{abstract}

Let $R$ be a ring with $1 \neq 0$ and $M$ an indecomposable unital right $R$-module of finite composition length. Let $S=\operatorname{End}\left(M_{R}\right)$ be the ring of endomorphisms of $M$ acting on the left. A result due to Fitting (see Faith [1, Corollary 17.17']) gives that $S$ is a local ring with its Jacobson radical nilpotent. By taking $M=R_{R}$, for some local right artinian ring $R$, which is not left artinian, one immediately sees that $S$ need not be left artinian. No such simple example seems existing in literature, showing that $S$ need not be right artinian. In this note we give an example showing that $S$ need not be right artinian. It follows from [2, Proposition 1] that the ring of endomorphisms of a finite length quasi-injective module is left artinian; here we give an elementary proof of this result. For the terms and result used in this note we refer to Faith [1].

The following theorem provides the example.

THEOREM 1. There exists a ring $R$ and a faithful right $R$-module $M$ such that

(i) $R$ is local, left artinian but not right artinian,

(ii) $M$ is a uniserial module having composition length 2,

(iii) $\operatorname{End}\left(M_{R}\right) \approx R$.

Proof. Let $K$ be a field and $F=K\left(X_{i}\right)$ be the field of fractions over $F$, in an infinite set of indeterminates $\left\{X_{i}\right\}_{i \in \Lambda}$. Let $\sigma: F \rightarrow F$ be the $K$-endomorphism of the field $F$ such that $\sigma\left(X_{\alpha}\right)=X_{\alpha}^{2}$. Then

$$
\sigma(F)=K\left(X_{i}^{2}\right) \neq F .
$$

A linear basis of $F$ over the subfield $\sigma(F)$ is $B=\{1\} \cup B_{1}$, where $B_{1}$ consists of all monomials of the form $X_{i_{1}} X_{i_{2}} \cdots X_{i_{n}}$ where $n \geqslant 1$ and $i_{1}, i_{2}, \ldots, i_{n}$ are finitely many distinct members of $\Lambda$. Consider the ring $R=F \times F$ in which addition is componentwise and the multiplication is by the rule

$$
(\alpha, \beta)(\gamma, \delta)=(\alpha \gamma, \alpha \delta+\sigma(\gamma) \beta) .
$$

Received by the editors May 22, 1984.

1980) Mathematics Subject Classification. Primary 16A65; Secondary 16A05.

Key nords and phrases. Uniserial modules, ring of endomorphisms, quasi-injective, module, artinian ring. ficld of fractions.

1985 American Mathematical Society $0002-9939 / 85 \$ 1.00+\$ .25$ per page 
$R$ is a local ring with maximal ideal $J=0 \times F$. Further ${ }_{R} R$ has composition length 2 , and $R$ is not right artinian. Let $G$ be the $\sigma(F)$-subspace of $F$ spanned by $B_{1}$. Then $A=0 \times G$ is a right ideal of $R$. As $G$ is a maximal $\sigma(F)$-subspace of $F, M=R / A$ is a uniserial right $R$-module of composition length 2. Consider the idealizer of $A$,

$$
I=I(A)=\{r \in R: r A \subset A\} .
$$

We have $S=\operatorname{End}(M) \approx I / A$. It is easy to see that

$$
I=\{(\alpha, \beta): \alpha G \subset G\} .
$$

We now show that $\sigma(F)=T$, where $T=\{\alpha \in F: \alpha G \subset G\}$. Trivially $\sigma(F) \subset T$. Take any $\alpha \in F \backslash \sigma(F)$. Then $\alpha=\alpha_{0}+\sum_{b \in B_{1}} \alpha_{b} b$ where $\alpha_{0} \in \sigma(F), \alpha_{b} \in \sigma(F)$, such that only finitely many of these coefficients are nonzero. As $\alpha \notin \sigma(F)$, for some $b \in B_{1}, \alpha_{b} \neq 0$. Fix a $b_{0} \in B_{1}$ with $\alpha_{b_{0}} \neq 0$. Consider any $b \in B_{1}$ such that $b \neq b_{0}$. We can write

$$
b_{0}=c X_{j_{1}} X_{j_{2}} \cdots X_{j_{r}}, \quad b=c X_{k_{1}} X_{k_{2}} \cdots X_{k_{s}},
$$

where $c$ is the largest degree monomial in $X_{i}$ 's dividing $b_{0}$ and $b$ both. Then by definition of $B_{1}$, the indices $j_{1}, j_{2}, \ldots, j_{r}, k_{1}, k_{2}, \ldots, k_{s}$ are all distinct. Thus $c^{2} \in \sigma(F)$ and

$$
b_{0} b=c^{2} X_{j_{1}} X_{j_{2}} \cdots X_{j_{r}} X_{k_{1}} X_{k_{2}} \cdots X_{k_{s}} \in G .
$$

Further as $0 \neq \alpha_{b_{0}} b_{0}^{2} \in \sigma(F)$, we get

$$
\alpha b_{0}=\alpha_{b_{0}} b_{0}^{2}+\alpha_{0} b_{0}+\sum_{h \neq b_{0}} \alpha_{b}\left(b b_{0}\right) \notin G .
$$

Hence $\alpha \notin T$. This shows that $I=\sigma(F) \times F=\sigma(F) \times \sigma(F)+A$ and $I / A \approx$ $\sigma(F) \times \sigma(F) \approx R$. Hence $S=\operatorname{End}\left(M_{R}\right) \approx R$. This proves the theorem.

We now give an elementary proof of the following result, which, otherwise, is a special case of [2, Proposition 1].

THEOREM 2. Let $M_{R}$ be a quasi-injective module of finite composition length. Then $S=\operatorname{End}\left(M_{R}\right)$ is left artinian.

Proof. We prove the result by induction on the composition length $d(M)$ of $M$. If $d(M)=1, S$ is a division ring and the result holds. Let $d(M)>1$ and the result hold for all quasi-injective modules of composition length less than $d(M)$. Let $J(M)$ be the Jacobson radical of $M$. Then $J(M)$ is quasi-injective and $d(J(M))<d(M)$. So $T=\operatorname{End}(J(M))$ is left artinian.

Define homomorphism $\sigma: S \rightarrow T$ such that $\sigma(f)=f \mid J(M)$ for every $f \in S$. As $M$ is quasi-injective $\sigma$ is onto. Thus $S / \operatorname{Ker} \sigma \approx T$. Now $M / J(M)$ is completely reducible. We write $\bar{M}=M / J(M)=K_{1} \oplus K_{2} \oplus \cdots \oplus K_{n}$. Let $K=\operatorname{Socle}(M)$. Now $f \in$ Ker $\sigma$ if and only if $f(J(M))=0$. As $M / J(M)$ is completely reducible, we get $f \in \operatorname{Ker} \sigma$ if and only if $f(M) \subset K$. Thus

$$
\operatorname{Ker} \sigma=\operatorname{Hom}(M, K) \approx \operatorname{Hom}(\bar{M}, K) \approx \oplus \sum_{i=1}^{n} \operatorname{Hom}\left(K_{i}, K\right) .
$$


Consider any $K_{i}$. Consider any $f(\neq 0) \in \operatorname{Hom}\left(K_{i}, K\right)$. Now $f$ is one-to-one and $K$ is completely reducible. Thus we can find $g: K \rightarrow K_{i}$ such that $g f=I_{K_{i}}$. So, for any $h \in \operatorname{Hom}\left(K_{i}, K\right), h g f=h$. Now $h g: K \rightarrow K$ can be extended to a member $\lambda$ of $S$. This all gives $\operatorname{Hom}\left(K_{i}, K\right)=\operatorname{Hom}\left(K_{i},{ }_{s} K\right)={ }_{s}\left[\operatorname{Hom}\left(K_{i}, K\right)\right]$ is either simple or 0 . Hence ${ }_{S} \operatorname{Ker} \sigma$ is a finite direct sum of simple modules. As $S / \operatorname{Ker} \sigma$ is also left artinian, we get $S$ is left artinian.

REMARK (added in proof). The module $M_{R}$ constructed in Theorem 1 is quasiinjective but not injective.

\section{REFERENCES}

1. C. Faith, Algebra II, Ring theory, Springer-Verlag, Berlin and New York, 1976.

2. M. Harada and T. Ishii, On endomorphism rings of noetherian quasi-injective modules, Osaka J. Math. 9 (1972), 217-223.

Department of Mathematics, Panjab University, Chandigarh, 160014 India

Department of Mathematics, Kuwait University, P.O. Box 5969, Kuwait 